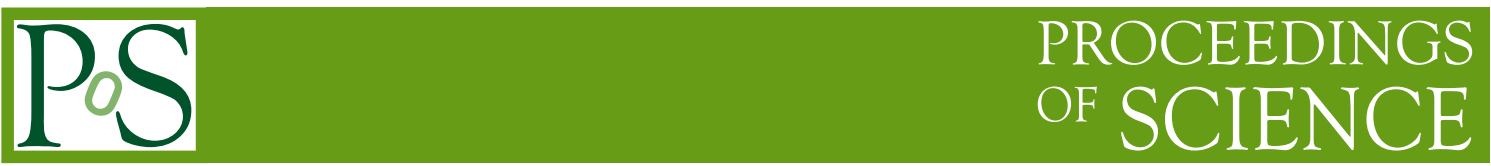

\title{
The LHCspin project
}

\author{
L. L. Pappalardo ${ }^{* a, b}$, V. Carassiti ${ }^{b}$, P. Di Nezza ${ }^{c}$, G. Ciullo $^{a, b}$, P. Lenisa ${ }^{a, b}$, E. Steffens ${ }^{d}$ \\ and A. Vasilyev ${ }^{e}$ \\ ${ }^{a}$ Dipartimento di Fisica e Scienze della Terra, Universitá di Ferrara, Italy \\ ${ }^{b}$ INFN Ferrara, Italy \\ ${ }^{c}$ INFN Laboratori Nazionali di Frascati, Frascati (Rome), Italy \\ ${ }^{d}$ Physics Dept., FAU Erlangen-Nürnberg, Erlangen, Germany \\ ePNPI Gatchina, Petersburg, Russia
}

E-mail: pappalardo@fe.infn.it, vitodfe.infn.it,

Pasquale.DiNezza@lnf.infn.it, ciullo@fe.infn.it, lenisa@fe.infn.it,

Erhard.Steffens@fau.de, Vasilyev_aa@pnpi.nrcki.ru

\begin{abstract}
The LHCspin project aims to bring both unpolarized and polarized physics at the LHC through the installation of a gaseous fixed target at the upstream end of the LHCb detector. The forward geometry of the LHCb spectrometer $(2<\eta<5)$ is perfectly suited for the reconstruction of particles produced in fixed-target collisions. The fixed-target configuration, with center-of-mass energies ranging from $\sqrt{s_{N N}}=72 \mathrm{GeV}$ in collisions with $\mathrm{Pb}$ beams to $\sqrt{s}=115 \mathrm{GeV}$ in pp interactions, allows to cover a wide backward center-of-mass rapidity region, corresponding to the poorly explored high $\mathrm{x}$-Bjorken and high $\mathrm{x}$-Feynman regimes. The project has several ambitious goals regarding heavy-ion physics and new-era quantitative searches in QCD through the study of the nucleon's internal dynamics in terms of both quarks and gluons degrees of freedom. In particular, the use of transversely polarized $\mathrm{H}$ and $\mathrm{D}$ targets will allow to study the quarks TMDs in pp collisions at unique kinematic conditions. Furthermore, being LHCb specifically designed for heavy-flavor physics, final states with c- or b-quarks (e.g. inclusive quarkonia production) will be efficiently reconstructed, thus providing, among other fundamental measurememnts, access to the so-far unknown gluons TMDs. The status of the project is presented along with a selection of physics opportunities.
\end{abstract}

XXVII International Workshop on Deep-Inelastic Scattering and Related Subjects - DIS2019

8-12 April, 2019

Torino, Italy

${ }^{*}$ Speaker. 


\section{Introduction}

Fixed-target pp and $\mathrm{pA}$ collisions with a proton beam at the $\mathrm{TeV}$ scale provide unique laboratories for the study of the nucleon's internal dynamics and, more in general, for the investigation of the complex phenomena arising in the non-perturbative regime of QCD. In particular, due to the substantial boost of the reaction products in the laboratory frame, fixed-target collisions measured with a forward spectrometer such as $\mathrm{LHCb}$, allow one to access the backward center-of-mass rapidity region $\left(-3<y^{*}<0\right)$, corresponding to the poorly explored high $x$-Bjorken regime. These measurements will thus allow to open the way to innovative and fundamental measurements in regions of the kinematic plane which are still essentially unexplored [1], Fig.1. Furthermore, the use of a gaseous target has the advantage of allowing for a broad variety of nuclear targets, thus providing novel probes for the study of the nucleon and nuclear structure, and for measurements of great interest ranging from heavy-ion physics to cosmic rays physics and dark matter search.
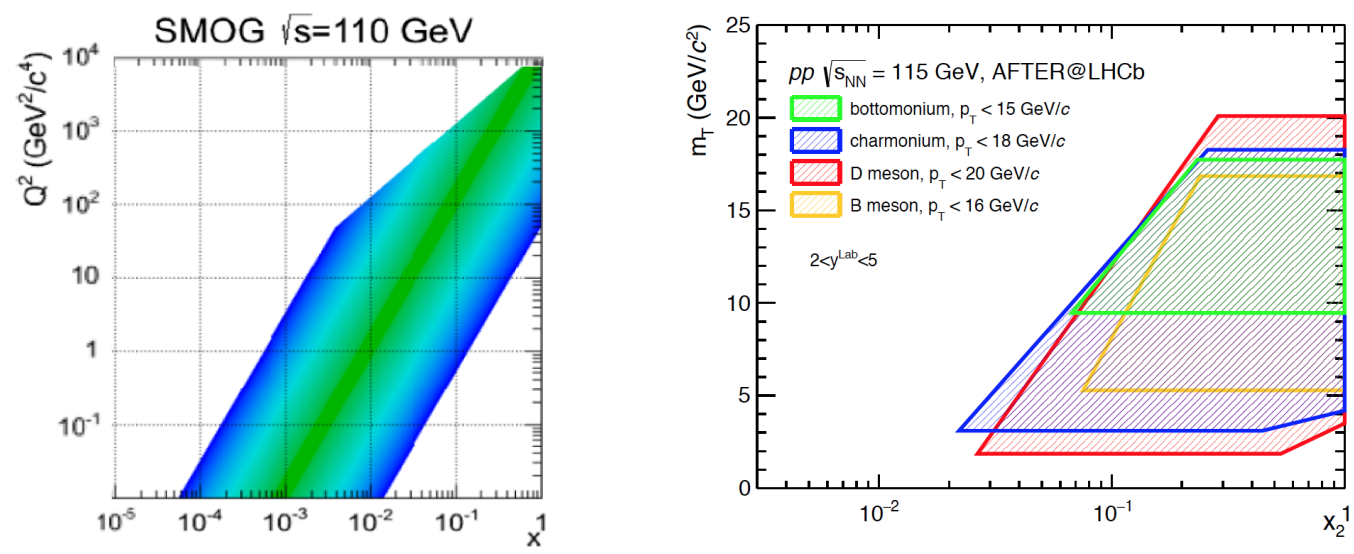

Figure 1: Left: Accessible range in the $\left(x, \mathrm{Q}^{2}\right)$ plane with fixed-target collisions at LHCb (SMOG) [2]. Right: Expected coverage in $x$-Bjorken for several processes including production of charmionium and bottomonium and $D$ and $B$ mesons within the LHCb acceptance $(2<\eta<5)[1]$.

\section{The physics case}

The physics case for a fixed-target program at the LHC is very rich and ambitious, and spans over many fundamental and complementary fields of high-energy physics: heavy-ion physics, nucleon structure, hadronic particle production, cosmic rays physics and dark matter search, etc. [2]. The first two subjects are discussed in some detail in the following sections.

\subsection{Heavy-ion physics}

Relativistic heavy-ion collisions allow to investigate the high-density and high-temperature regime of QCD. The production of heavy quarks is particularly suited for the study of the phase transition between ordinary hadronic matter and the Quark-Gluon Plasma (QGP). Since their masses are significantly higher than the QGP critical temperature $\left(T_{c} \sim 156 \mathrm{MeV}\right)$ they are produced in the 
early stages of the collision, and in a time scale that is shorter than that of QGP formation. A correct interpretation of their re-scattering and energy-loss mechanisms in the QGP medium requires a full understanding of the cold-nuclear-matter effects. Therefore, the study of heavy-meson production in proton-induced reactions on various nuclear targets, where QGP is not expected to be formed, is essential to establish a robust baseline for a correct interpretation of the QGP-induced suppression patterns observed in heavy-ion collisions. Furthermore, studying the production of quarkonia and heavy mesons with different nuclear targets will allow to set stringent constraints on the nuclear PDFs (nPDFs) and to investigate their peculiar features, such as the antishadowing effect at large $x$ and the EMC effect at very large $x$, whose origin is still under debate. Another interesting feature, which is also expected to be significant at large $x$, and thus potentially at reach in fixed-target collisions at the LHC, is the contribution of intrinsic (i.e. valence-like) charm to the ordinary charm-quark parton distributions [3]. Finally, by exploiting the use of the LHC Pb beams, one can also study QGP formation in fixed-target PbA collisions at $\sqrt{s_{N N}}=72 \mathrm{GeV}$ through the measurement of flow observables and correlations for a variety of collision systems. In particular, by measuring the production of different charmonia excited states $\left(J / \psi, \psi^{\prime}, \chi_{c}\right.$, etc. ) a study of the intriguing phenomenon of sequential suppression [4] is also possible.

\subsection{Nucleon structure}

The nucleon structure is traditionally parametrized in terms of PDFs, which, in their simplest (collinear) form, are functions of the longitudinal momentum fraction of quarks and gluons, expressed by the Bjorken- $x$ variable. Although tremendous advances have been made over the past decades in defining the quark and gluon dynamical substructure of the nucleon, the present knowledge of the PDFs still suffers from large uncertainties, especially at very-high and very-low $x$, leaving open fundamental questions about QCD and confinement. In many cases, the PDF uncertainties have become the limiting factor in the accuracy of the predictions for LHC measurements, especially concerning measurements of SM and BSM observables. Most of our knowledge about the collinear PDFs comes from decades of inclusive DIS experiments. Considering also the explicit dependence of PDFs on the parton transverse momenta, radically new perspectives in the exploration of the structure of the nucleon can be opened. Differently from the collinear PDFs, which only provide a 1-dimensional description of the nucleon structure in terms the longitudinal momentum fraction of partons, TMDs are sensitive to spin-orbit correlations inside the nucleon. They are thus indirectly sensitive to the still unknown parton orbital angular momentum, the main missing piece in the proton spin puzzle. In addition, they provide the possibility to map the parton densities in the 3-dimensional momentum space, spanned by the longitudinal momentum fraction $x$ and by the two transverse momentum components, allowing for a nucleon tomography in momentum space [5]. Quark TMDs have been experimentally explored in the last decades, mainly in semi-inclusive deep-inelastic scattering processes. The same distributions can now be studied at LHC in fixed-target hadron-hadron collision (Drell-Yan) at unique kinematic conditions, providing a complementary approach and an ideal test-bench for testing universality, factorization and evolution of QCD. In contrast to the quark TMDs, the experimental access to the gluon TMDs is still extremely limited. In high-energy hadronic collisions, heavy quarks are dominantly generated by gluon-gluon interactions. As a consequence, the most efficient way to access the gluon distributions is through the study of inclusive heavy-flavour production in fixed-target measurements 
at $\sqrt{s}=115$. Among the gluon TMDs, particularly interesting is the spin-dependent gluon Sivers function (GSF), which maps the distribution of unpolarized gluons inside a transversely polarized proton. Transverse single-spin asymmetries (TSSAs) in inclusive quarkonium production in fixedtarget $p p$ interactions turns out to be the ideal observable to have access to the GSF and other spin-dependent gluon TMDs. More precisely, since transverse-momentum-dependent QCD factorization requires $p_{T}(Q) \ll M_{Q}$, where $Q$ denotes a heavy quark, the safest processes to be studied with a polarized hydrogen target are back-to-back production of quarkonia and isolated photons or associated quarkonium production, where only the relative $p_{T}$ has to be small compared to $M_{Q}$.

\section{Unpolarized Target}

Among the main LHC experiments, LHCb is the only detector that can already run both in collider and fixed-target mode. The LHCb fixed-target system, called SMOG (System for Measuring the Overlap with Gas), was originally conceived for precise luminosity determinations through the beam-gas imaging technique [6]. The SMOG system allows to inject a low flow rate of noble gas into the vacuum vessel of the LHCb VErtex LOcator (VELO) detector. A temporary local pressure bump of about $10^{-7}$ mbar is obtained in the LHCb beam-pipe section (over a length of about 40 $\mathrm{m}$ around the nominal interaction point), which is about two order of magnitude higher than the nominal LHC vacuum pressure and one order of magnitude lower than the LHC vacuum interlock limit. The resulting beam-gas collision rate allows for a precise determination of the beam density profiles. As an additional important feature, SMOG gives the unique opportunity to operate $\mathrm{LHCb}$ in fixed target mode, and to study proton-nucleus and nucleus-nucleus collisions on various target types and at different center-of-mass energies. Several dedicated runs have already been performed since 2015 using $\mathrm{He}$, Ar, or Ne targets with proton and $\mathrm{Pb}$ beams, and first interesting physics results have recently been published [7, 8]. The system is now being upgraded (SMOG2) with the implementation of a storage cell for the target gas $[9,10]$ to be installed at the upstream edge of the VELO, coaxial with the LHC beam. This new setup has the major advantage of increasing by up to two orders of magnitude the target areal density (and thus the luminosity), by injecting the same amount of gas of SMOG. Other important advantages are the implementation of a more sophisticated gas-feed system with multiple injection lines which will allow for a precise (at few percent-level) determination of the target density and for the injection of additional gas species $(\mathrm{H}$, $\mathrm{D}, \mathrm{N}, \mathrm{O}, \mathrm{Kr}$ and $\mathrm{Xe}$ ), a well defined interaction region (limited by the cell length, $20 \mathrm{~cm}$ ), and the possibility to run in parallel (i.e. simultaneously) with the collider mode, with a negligible impact on the beam life-time and on the LHCb mainstream physics program. The final design of the storage cell and its arrangement inside the VELO vessel are shown in Fig. 2. Possible scenarios for the integrated luminosities to be collected with SMOG2 using different gas targets during the 3 years of Run3 data taking are reported in Ref. [2].

\section{Polarized Target}

The realization of an important part of the broad physics program described above requires the implementation of a polarized gaseous target at the upstream end of the LHCb spectrometer [11]. This device, together with the upgraded SMOG system (SMOG2), constitutes the core 

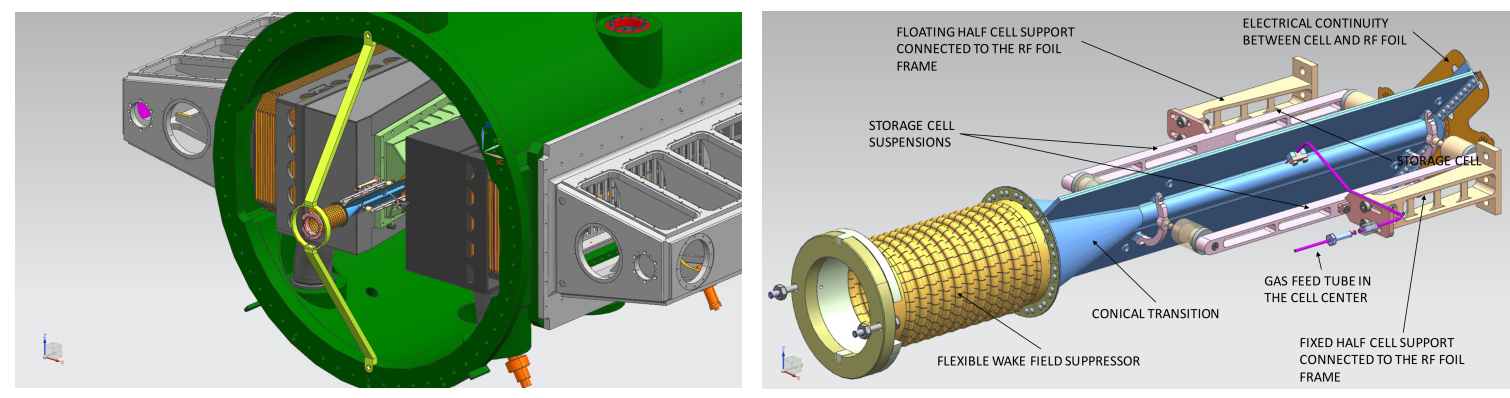

Figure 2: Left: SMOG2 storage cell mounted inside LHCb, in front of the VELO detector. Right: details of the storage cell.

of the LHCspin project [12]. The concept of the apparatus is based on the polarized target system used at the HERMES experiment (DESY) [13]. The setup will consist of four main components: an Atomic Beam Source (ABS), a Target Chamber (TC) hosting a T-shaped storage cell with a length of the order of $30 \mathrm{~cm}$, a diagnostic system, and an additional tracking detector. The ABS generates a beam of polarized atomic gas ( $\mathrm{H}$ or $\mathrm{D})$ that is injected into a storage cell, in order to maximize the target areal density. The cell is placed inside the target chamber, into the LHC primary vacuum. The diagnostic system, including a Breit-Rabi polarimeter and a Gas Target Analyzer (TGA), allows to monitor both the fraction of atomic gas into the cell and the degree of polarization. The target chamber also hosts a transverse magnet $(\sim 300 \mathrm{mT})$, needed to define and keep the transverse polarization of the target gas. The HERMES polarized target has been successfully operated over a decade, with very high performances [13]. However, due to the limited space available upstream of the LHCb spectrometer, a new, more compact system has to be designed and constructed. The arrangement of the Polarized Gas Target (PGT) in the beam line upstream of the VELO is shown schematically in Fig.3. Due to the distance between the target cell and the VELO detector, an additional tracker has to be installed inside the TC, in order to supplement the tracking capabilities of the VELO in this upstream region. To maximize the acceptance, this new tracker must be located as close as possible to the beam. As a consequence, radiation hardness and high granularity are mandatory. Considering the geometry of the cell, assuming an ABS intensity of $6.5 \cdot 10^{16}$ atoms/s into the cell feed tube and a (conservative) LHC proton beam intensity of $3.8 \cdot 10^{18} \mathrm{p} / \mathrm{s}$ for the LHC Run4, one obtains an instantaneous luminosity for $\mathrm{pH}$ collisions of the order of $L_{p H}=2.7 \cdot 10^{32} \mathrm{~cm}^{-2} s^{-1}$ with the cell at room temperature, or $L_{p H}=4.6 \cdot 10^{32} \mathrm{~cm}^{-2} \mathrm{~s}^{-1}$ with the cell cooled at $100 \mathrm{~K}$.

\section{Conclusions}

Fixed target collisions at the LHC offer a unique opportunity for a laboratory for QCD and astroparticle in unexplored kinematic regions. The upgraded LHCb unpolarized gas target, SMOG2, will be installed by the end of 2019. LHCspin aims at installing a polarized gas target in front of the LHCb spectrometer during the LHC LS3. If approved, the latter setup will bring for the first time spin physics to the LHC, and $\mathrm{LHCb}$ will become the first experiment simultaneously running in collider and fixed-target mode with polarized targets, opening a whole new range of explorations. 


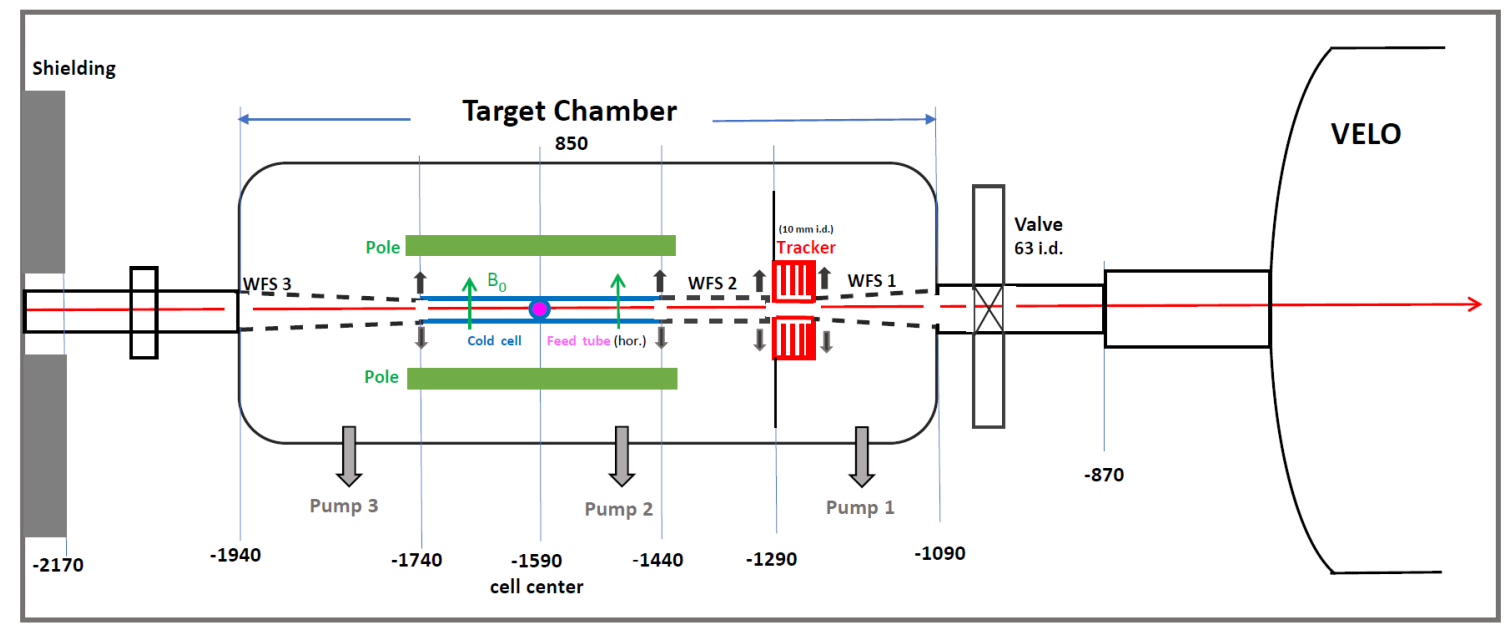

Figure 3: Schematic drawing of the proposed PGT arrangement upstream of VELO detector.

\section{References}

[1] C. Hadjidakis at al., A Fixed-Target Programme at the LHC: Physics Case and Projected Performances for Heavy-Ion, Hadron, Spin and Astroparticle Studies, arXiv:1807.00603 [hep-ex].

[2] PBC Report, Physics opportunities with the fixed target program of the LHCb experiment using an unpolarized gas target, LHCb-PUB-2018-015.

[3] J. Pumplin et al., Charm parton content of the nucleon, Phys. Rev. D 75, 054029 (2007).

[4] H. Satz, Colour Deconfinement and Quarkonium Binding, J. Phys. G32:R25, 2006, arXiv:hep-ph/0512217.

[5] A. Bacchetta et al., Extraction of partonic transverse momentum distributions from semi-inclusive deep-inelastic scattering, Drell-Yan and Z-boson production, JHEP 06 (2017) 081

[6] C. Barschel, Precision luminosity measurements at LHCb with beam-gas imaging, CERN-THESIS-2013-301, RWTH Aachen University, 2014.

[7] LHCb collaboration, R. Aaij et al., Measurement of antiproton production in pHe collisions at $\sqrt{s_{N N}}$ = 110 GeV, Phys. Rev. Lett. 121, 222001 (2018), arXiv:1808.06127.

[8] LHCb collaboration, R. Aaij et al., First Measurement of Charm Production in its Fixed-Target Configuration at the LHC, Phys. Rev. Lett. 122, 132002 (2019), arXiv:1810.07907v2.

[9] V.Carassiti et al., SMOG2 Technical Proposal, CERN-PBC-Notes-2018-007, https://cds.cern.ch/record/2651269

[10] LHCb collaboration, SMOG2 Technical Design Report, https://cds.cern.ch/record/2673690/files/LHCB-TDR-020.pdf

[11] E. Steffens, Design Considerations of a Polarized Gas Target for the LHC, Proceeding of Science (SPIN2018), https://pos.sissa.it/, PoS(SPIN2018)098.

[12] C. A. Aidala et al., The LHCspin project, CERN-ESPP-Note-2018-111, arXiv:1901.08002.

[13] HERMES Collaboration, A. Airapetian et. al., The HERMES polarized hydrogen and deuterium gas target in the HERA electron storage ring, Nucl. Instrum. Meth. A540 (2005) 68-101, [physics/0408137]. 